\title{
THE USE OF THE GENERALIZED SINC-GAUSSIAN SAMPLING FOR NUMERICALLY COMPUTING EIGENVALUES OF PERIODIC DIRAC SYSTEM*
}

\author{
RASHAD M. ASHARABI ${ }^{\dagger}$ AND MOHAMMED M. THARWAT ${ }^{\ddagger}$
}

\begin{abstract}
The generalized sinc-Gaussian sampling operator is established by Asharabi (2016) to approximate two classes of analytic functions. In this paper, we use this operator to construct a new sampling method to approximate the eigenvalues of the periodic (semi-periodic) Dirac system of differential equations problem. The convergence rate of this method is of exponential type, i.e., $\mathrm{e}^{-\alpha_{r} N} / \sqrt{N}, \alpha_{r}=((r+1) \pi-\sigma h) / 2$. The sinc-Gaussian and Hermite-Gauss methods are special cases of this method. We estimate the amplitude error associated to this operator, which gives us the possibility to establish the error analysis of this method. Various illustrative examples are presented and they show a good agreement with our theoretical analysis.
\end{abstract}

Key words. Generalized sinc-Gaussian, eigenvalues, periodic Dirac system, convergence rate.

AMS subject classifications. 30E10, 34L15, 47E05, 41A25, 94A20, 41A80.

1. Introduction. Let $B_{\sigma}^{p}$, for $\sigma>0$ and $1 \leq p \leq \infty$, be the Bernstein space of all entire functions $f: \mathbb{C} \rightarrow \mathbb{C}$ that belong to $L^{2}(\mathbb{R})$, when restricted to the real axis, and are of exponential type $\sigma$, so that they satisfy the inequality $|f(z)| \leq A \exp (\sigma|\Im z|)$, with $A>0$ and $z \in \mathbb{C}$. The Bernstein spaces satisfies the following inclusions (cf. [16])

$$
B_{\sigma}^{1} \subset B_{\sigma}^{p} \subset B_{\sigma}^{q} \subset B_{\sigma}^{\infty}, \quad 1<p<q<\infty .
$$

Asharabi defines in [4] the generalized sinc-Gaussian operator $\mathcal{G}_{r, h, N}: B_{\sigma}^{\infty} \rightarrow B_{\sigma}^{\infty}$ as follows:

$$
\begin{aligned}
& \mathcal{G}_{r, h, N}[f](z):= \\
& \sum_{n \in \mathbb{Z}_{N}(z)} \sum_{i+j+k+l=r} f^{(i)}(n h) P_{r+k-j}(z) \operatorname{sinc}^{r+1}\left(\pi h^{-1} z-n \pi\right) e^{-\frac{\alpha_{r}}{N}\left(h^{-1} z-n\right)^{2}},
\end{aligned}
$$

where $\alpha_{r}:=((r+1) \pi-h \sigma) / 2, \mathbb{Z}_{N}(z):=\left\{n \in \mathbb{Z}:\left|\left\lfloor h^{-1} \Re z+1 / 2\right\rfloor-n\right| \leq N\right\}, r \in \mathbb{N}_{\circ}$, $z \in \mathbb{C}, h \in(0,(r+1) \pi / \sigma]$, and $N \in \mathbb{Z}^{+}$. The symbol $\lfloor x\rfloor$ denotes the integer part of $x$. The sinc function is defined by

$$
\operatorname{sinc}(t):= \begin{cases}\frac{\sin t}{t}, & t \neq 0 \\ 1, & t=0\end{cases}
$$

Here $P_{r+k-j}$ is a polynomial of degree $r+k-j$ which is defined by

$$
P_{r+k-j}(z):=\beta_{i, j, k, l}\left(h^{-1} z-n\right)^{r-j} H_{k}\left(\frac{\sqrt{\alpha_{r}}(z-n h)}{\sqrt{N} h}\right),
$$

where $H_{k}(z)$ is the $k$-th degree Hermite polynomial

$$
H_{k}(z):=(-1)^{k} \exp \left(z^{2}\right) \frac{d^{k}}{d z^{k}} \exp \left(-z^{2}\right)=\sum_{m=0}^{\lfloor k / 2\rfloor} \frac{(-1)^{m} k !(2 z)^{k-2 m}}{m !(k-2 m) !},
$$

*Received July 10, 2018. Accepted September 2, 2018. Published online on November 14, 2018. Recommended by Frank Stenger.

${ }^{\dagger}$ Department of Mathematics, College of Arts and Sciences, Najran University, Najran, Saudi Arabia (rashad1974@hotmail.com).

${ }^{\ddagger}$ Department of Mathematics, Faculty of Science, Beni-Suef University, Beni-Suef, Egypt (zahraa26@yahoo.com). 


\section{ETNA}

and the constant $\beta_{i, j, k, l}$ is given by

$$
\beta_{i, j, k, l}:=\frac{\pi^{r+1}(-1)^{(r+1) n+k} h^{i}}{i ! k ! l !}\left[\frac{d^{l}}{d \zeta^{l}}\left(\frac{\zeta-n}{\sin (\pi \zeta)}\right)^{r+1}\right]_{\zeta=n} .
$$

In fact, operator (1.1) was introduced for wider classes than Bernstein spaces, but in this study we restrict this operator only to functions from the Bernstein space $B_{\sigma}^{\infty}$. This operator is produced from the modification of the generalized Hermite sampling with Gaussian function $e^{-x^{2}}$. The modification of the classical sampling series using a Gaussian multiplier goes back to Qian and his co-authors; cf. [11,12]. The generalized Hermite sampling was introduced in the literature; cf. $[8,14,15]$. We remark that a particular case of $(1.1)$, when $r=0$, has been investigated by Schmeisser and Stenger in [13]. This case is called sinc-Gaussian sampling and it has been extensively used for approximating eigenvalues of various boundary value problems. By methods similar to those developed in [13], Asharabi and Prestin introduced the Hermite-Gauss operator, which is a modification of Hermite sampling with a Gaussian function; see [6]. This case is included in the operator (1.1) as a special case when $r=1$, and it has been used for approximating eigenvalues of boundary value problems; cf. [5]. The history of the sampling methods, which approximate the eigenvalues of the boundary value problems, has been introduced in [7].

In [4, Corollary 3.5], the author investigated a bound for approximating functions from the Bernstein space $B_{\sigma}^{\infty}$ by the generalized sinc-Gaussian operator, and proved that if $f \in B_{\sigma}^{\infty}$, then we have for all $x \in \mathbb{R}$,

$$
\left|f(x)-\mathcal{G}_{r, h, N}[f](x)\right| \leq 2^{r}\left|\sin ^{r+1}\left(h^{-1} \pi x\right)\right|\|f\|_{\infty} \frac{e^{-\alpha_{r} N}}{\pi \sqrt{\alpha_{r} N}} .
$$

The convergence rate of this operator is of exponential type, i.e., $e^{-\alpha_{r} N} / \sqrt{N}$, with $\alpha_{r}$ defined as above. It is clear that the precision increases when $N$ and $h$ are fixed and $r$ increases, with the additional cost that the samples $f^{(i)}(n h), i=1, \ldots, r$, need to be calculated. Moreover, the precision increases when $N$ and $r$ are fixed and $h$ decreases, without any additional cost except that the function is approximated on a smaller domain.

Our objective is to use the generalized sinc-Gaussian sampling operator $\mathcal{G}_{r, h, N}$ for approximating the eigenvalues of the Dirac system, which consists of the system of differential equations

$$
\begin{aligned}
u_{2}^{\prime}(x, \lambda)+p_{1}(x) u_{1}(x, \lambda) & =\lambda u_{1}(x, \lambda), \\
-u_{1}^{\prime}(x, \lambda)+p_{2}(x) u_{2}(x, \lambda) & =\lambda u_{2}(x, \lambda),
\end{aligned}
$$

with $x \in[0, a]$ and boundary conditions

$$
u_{1}(0, \lambda)=(-1)^{\ell-1} u_{1}(a, \lambda), \quad u_{2}(0, \lambda)=(-1)^{\ell-1} u_{2}(a, \lambda), \quad \ell=1,2,
$$

where $\lambda \in \mathbb{C}$ and $p_{1}(\cdot)$ and $p_{2}(\cdot)$ are real-valued smooth periodic functions with period $a$. For $\ell=1$, the boundary value problem (1.5)-(1.6) is said to be periodic, while, when $\ell=2$, the problem (1.5)-(1.6) is said to be semi-periodic. The spectral theory for the problem (1.5)(1.6) is established in [10]. The problem has a denumerable set of real eigenvalues (cf. [9, $10]$ ), and its eigenvalues may repeat with multiplicity not exceeding two; cf. [10, pp. 202204]. Up to now, the generalized sinc-Gaussian sampling operator $\mathcal{G}_{r, h, N}, r \geq 2$, has not been used for approximating the eigenvalues of boundary value problems. Moreover, to the best of our knowledge, the approximating eigenvalues of various Dirac systems has been 
investigated using different sampling methods (cf. [2,3]), except the periodic (semi-periodic) Dirac system (1.5)-(1.6). The approximating eigenvalues of problem (1.5)-(1.6) were not investigated before using any sampling methods.

The rest of the paper is organized as follows: the next section is devoted to establish the method. The error analysis associated with our method is given in Section 3. Section 4 deals with various illustrative examples, which show the efficiency and accuracy of this method. Lastly, Section 5 concludes the paper.

2. The method. This section is devoted to the construction of our method. This method approximates the eigenvalues of problem (1.5)-(1.6) using the generalized sampling operator (1.1). Let $\varphi_{\ell}(\cdot, \lambda)=\left(\varphi_{\ell 1}(\cdot, \lambda), \varphi_{\ell 2}(\cdot, \lambda)\right)^{\top}$ and $\vartheta_{\ell}(\cdot, \lambda)=\left(\vartheta_{\ell 1}(\cdot, \lambda), \vartheta_{\ell 2}(\cdot, \lambda)\right)^{\top}$ be two solutions of (1.5) satisfying the following initial conditions

$$
\varphi_{\ell 1}(0, \lambda)=\vartheta_{\ell 2}(0, \lambda)=0, \quad \varphi_{\ell 2}(0, \lambda)=\vartheta_{\ell 1}(0, \lambda)=(-1)^{\ell-1}, \quad \ell=1,2,
$$

where $A^{\top}$ denotes the transpose of a matrix $A$. From now on, unless otherwise stated, $\ell=1,2$. The solutions $\varphi_{\ell i}(\cdot, \lambda)$ and $\vartheta_{\ell i}(\cdot, \lambda), i, \ell=1,2$, are entire functions in $\lambda$; cf. [9, 10]. The eigenvalues of the problem (1.5)-(1.6) are the zeros of the characteristic function (cf. [10, p. 203])

$$
\Delta_{\ell}(\lambda)=\varphi_{\ell 2}(a, \lambda)+\vartheta_{\ell 1}(a, \lambda)+(-1)^{\ell} 2,
$$

which is an entire function in $\lambda$. The function $\Delta_{1}(\lambda)$ is the characteristic function of the periodic problem, while $\Delta_{2}(\lambda)$ is the characteristic function of the semi-periodic problem. In [10, p. 204], the authors introduced a necessary and sufficient condition for the zero of (2.2) to be multiple. They proved that a point $\lambda^{*}$ is a multiple zero of (2.2) if and only if

$$
\varphi_{\ell 1}\left(a, \lambda^{*}\right)=\vartheta_{\ell 2}\left(a, \lambda^{*}\right)=0 .
$$

We will use this condition in the examples of Section 4 to show whether a zero of $\Delta_{\ell}(\lambda)$ is double or not. The main idea of this method is to construct an entire function, which will be denoted by $\widetilde{\Delta}_{\ell, N}(\lambda)$, using the generalized sampling operator (1.1). This function will be very close to the characteristic function $\Delta_{\ell}(\lambda)$. Therefore, the zeros of this function will be accurate approximations to the zeros of the characteristic function $\Delta_{\ell}(\lambda)$.

Applying similar methods to those developed by Levitan and Sargsjan in [9, p. 220], $\varphi_{\ell}(\cdot, \lambda)$ and $\vartheta_{\ell}(\cdot, \lambda)$ satisfy the system of integral equations

$$
\begin{aligned}
& \varphi_{\ell 1}(x, \lambda)=(-1)^{\ell} \sin \lambda x+\mathcal{T}_{1} \varphi_{\ell 1}(x, \lambda)+\widetilde{\mathcal{T}}_{2} \varphi_{\ell 2}(x, \lambda), \\
& \varphi_{\ell 2}(x, \lambda)=(-1)^{\ell-1} \cos \lambda x-\widetilde{\mathcal{T}}_{1} \varphi_{\ell 1}(x, \lambda)+\mathcal{T}_{2} \varphi_{\ell 2}(x, \lambda), \\
& \vartheta_{\ell 1}(x, \lambda)=(-1)^{\ell-1} \cos \lambda x+\mathcal{T}_{1} \vartheta_{\ell 1}(x, \lambda)+\widetilde{\mathcal{T}}_{2} \vartheta_{\ell 2}(x, \lambda), \\
& \vartheta_{\ell 2}(x, \lambda)=(-1)^{\ell-1} \sin \lambda x-\widetilde{\mathcal{T}}_{1} \vartheta_{\ell 1}(x, \lambda)+\mathcal{T}_{2} \vartheta_{\ell 2}(x, \lambda),
\end{aligned}
$$

where $\mathcal{T}_{i}$ and $\widetilde{\mathcal{T}}_{i}, i=1,2$, are Volterra operators defined by

$$
\mathcal{T}_{i} u(x, \lambda):=\int_{0}^{x} \sin \lambda(x-t) p_{i}(t) u(t, \lambda) d t, \quad \widetilde{\mathcal{T}}_{i} u(x, \lambda):=\int_{0}^{x} \cos \lambda(x-t) p_{i}(t) u(t, \lambda) d t .
$$

For the sake of convenience, we define $f_{\ell i}(\cdot, \lambda), i=1, \ldots, 4$, to be

$$
\begin{array}{ll}
f_{\ell 1}(x, \lambda):=\mathcal{T}_{1} \varphi_{\ell 1}(x, \lambda)+\widetilde{\mathcal{T}}_{2} \varphi_{\ell 2}(x, \lambda), & f_{\ell 2}(x, \lambda):=-\widetilde{\mathcal{T}}_{1} \varphi_{\ell 1}(x, \lambda)+\mathcal{T}_{2} \varphi_{\ell 2}(x, \lambda), \\
f_{\ell 3}(x, \lambda):=\mathcal{T}_{1} \vartheta_{\ell 1}(x, \lambda)+\widetilde{\mathcal{T}}_{2} \vartheta_{\ell 2}(x, \lambda), \quad f_{\ell 4}(x, \lambda):=-\widetilde{\mathcal{T}}_{1} \vartheta_{\ell 1}(x, \lambda)+\mathcal{T}_{2} \vartheta_{\ell 2}(x, \lambda) .
\end{array}
$$


LEMma 2.1. The functions $f_{\ell i}(x, \lambda), \ell=1,2, i=2,3$, are entire in $\lambda$ for any fixed $x \in[0, a]$ and satisfy the growth condition

$$
\left|f_{\ell i}(x, \lambda)\right| \leq \eta \exp (\eta) e^{|\Im \lambda| x}, \quad \lambda \in \mathbb{C},
$$

where

$$
\eta:=\int_{0}^{a}\left(\left|p_{1}(t)\right|+\left|p_{2}(t)\right|\right) d t .
$$

Proof. Since $f_{\ell 1}(x, \lambda):=\mathcal{T}_{1} \varphi_{\ell 1}(x, \lambda)+\widetilde{\mathcal{T}}_{2} \varphi_{\ell 2}(x, \lambda)$, then from (2.4) and (2.5) we obtain

$$
f_{\ell 1}(x, \lambda)=(-1)^{\ell} \mathcal{T}_{1} \sin \lambda x+(-1)^{\ell-1} \widetilde{\mathcal{T}}_{2} \cos \lambda x+\mathcal{T}_{1} f_{\ell 1}(x, \lambda)+\widetilde{\mathcal{T}}_{2} f_{\ell 2}(x, \lambda) .
$$

Using the inequalities

$$
|\sin z| \leq e^{|\Im z|}, \quad|\cos z| \leq e^{|\Im z|}, \quad z \in \mathbb{C},
$$

leads for $\lambda \in \mathbb{C}$ to

$$
\begin{aligned}
\left|f_{\ell 1}(x, \lambda)\right| \leq & \left|\mathcal{T}_{1} \sin \lambda x\right|+\left|\widetilde{\mathcal{T}}_{2} \cos \lambda x\right|+\left|\mathcal{T}_{1} f_{\ell 1}(x, \lambda)\right|+\left|\widetilde{\mathcal{T}}_{2} f_{\ell 2}(x, \lambda)\right| \\
\leq & e^{|\Im \lambda| x} \int_{0}^{x}\left[\left|p_{1}(t)\right|+\left|p_{2}(t)\right|\right] d t \\
& +e^{|\Im \lambda| x} \int_{0}^{x}\left[\left|p_{1}(t)\right|\left|f_{\ell 1}(t, \lambda)\right|+\left|p_{2}(t)\right|\left|f_{\ell 2}(t, \lambda)\right|\right] e^{-|\Im \lambda| t} d t \\
\leq & \eta e^{|\Im \lambda| x}+e^{|\Im \lambda| x} \int_{0}^{x}\left[\left|p_{1}(t)\right|\left|f_{\ell 1}(t, \lambda)\right|+\left|p_{2}(t)\right|\left|f_{\ell 2}(t, \lambda)\right|\right] e^{-|\Im \lambda| t} d t .
\end{aligned}
$$

The above inequality can be reduced to

$$
e^{-|\Im \lambda| x}\left|f_{\ell 1}(x, \lambda)\right| \leq \eta+\int_{0}^{x}\left[\left|p_{1}(t)\right|\left|f_{\ell 1}(t, \lambda)\right|+\left|p_{2}(t)\right|\left|f_{\ell 2}(t, \lambda)\right|\right] e^{-|\Im \lambda| t} d t
$$

Similarly, we can prove that

$$
e^{-|\Im \lambda| x \mid}\left|f_{\ell 2}(x, \lambda)\right| \leq \eta+\int_{0}^{x}\left[\left|p_{1}(t)\right|\left|f_{\ell 1}(t, \lambda)\right|+\left|p_{2}(t)\right|\left|f_{\ell 2}(t, \lambda)\right|\right] e^{-|\Im \lambda| t} d t
$$

Then from (2.10), (2.11), and Lemma 3.1 of [10, pp. 204], we obtain

$$
\left|f_{\ell 2}(x, \lambda)\right| \leq \eta \exp (\eta) e^{|\Im \lambda| x} .
$$

From (2.6), (2.7), (2.9), and by applying the same procedure as in the cases of $\left|f_{\ell 1}(x, \lambda)\right|$ and $\left|f_{\ell 2}(x, \lambda)\right|$, we can conclude

$$
\left|f_{\ell 3}(x, \lambda)\right| \leq \eta \exp (\eta) e^{|\Im \lambda| x}
$$

We split the characteristic function $\Delta_{\ell}(\lambda)$ into two parts, one is known and the other is not, but it belongs to the Bernstein space $B_{a}^{\infty}$, i.e.,

$$
\Delta_{\ell}(\lambda):=\mathcal{K}_{\ell}(\lambda)+\mathcal{U}_{\ell}(\lambda),
$$


where $\mathcal{U}_{\ell}(\lambda)$ is the unknown part involving the integral operators

$$
\mathcal{U}_{\ell}(\lambda):=f_{\ell 2}(a, \lambda)+f_{\ell 3}(a, \lambda),
$$

and $\mathcal{K}_{\ell}(\lambda)$ is the known part

$$
\mathcal{K}_{\ell}(\lambda):=2\left((-1)^{\ell-1} \cos a \lambda+(-1)^{\ell}\right) .
$$

LeMma 2.2. The function $\mathcal{U}_{\ell}(\lambda), \ell=1,2$, belongs to $B_{a}^{\infty}$.

Proof. Using (2.13) and Lemma 2.1, the function $\mathcal{U}_{\ell}(\lambda)$ is entire in $\lambda$ and satisfies the following estimate

$$
\left|\mathcal{U}_{\ell}(\lambda)\right| \leq 2 \eta \exp (\eta) e^{|\Im \lambda| a}
$$

where $\eta$ is defined in (2.8). Therefore $\mathcal{U}_{\ell}(\lambda) \in B_{a}^{\infty}$.

Now, we can approximate $\mathcal{U}_{\ell}(\lambda)$ using the generalized sinc-Gaussian sampling (1.1) to get an approximation of $\Delta_{\ell}(\lambda)$, which will be denoted by $\Delta_{\ell, N}(\lambda)$, i.e.,

$$
\Delta_{\ell}(\lambda) \approx \Delta_{\ell, N}(\lambda):=\mathcal{K}_{\ell}(\lambda)+\mathcal{G}_{r, h, N}\left[\mathcal{U}_{\ell}\right](\lambda),
$$

where $h=(0,(r+1) \pi / a]$ and $\alpha_{r}=((r+1) \pi-h a) / 2$. In view of (2.12), the samples of the operator $\mathcal{G}_{r, h, N}$ are given by

$$
\mathcal{U}_{\ell}^{(i)}(n h):=\Delta_{\ell}^{(i)}(n h)-\mathcal{K}_{\ell}^{(i)}(n h), \quad i=0, \ldots, r,
$$

where $n \in \mathbb{Z}_{N}(\lambda)$, which is defined above. Unfortunately, those samples cannot be computed explicitly in the general case. Then, we compute those samples numerically, and this makes the amplitude error appear. Indeed, the amplitude error arises when the exact values $\mathcal{U}_{\ell}^{(i)}(n h)$, $i=0, \ldots, r$, of the operator $\mathcal{G}_{r, h, N}\left[\mathcal{U}_{\ell}\right]$ are replaced by approximations. Let $\tilde{\mathcal{U}}_{\ell}(n h)$ be the approximation of the samples $\mathcal{U}_{\ell}(n h):=\mathcal{U}_{\ell}^{(0)}(n h)$

$$
\tilde{\mathcal{U}}_{\ell}(n h)=\varphi_{\ell 2}(a, n h)+\vartheta_{\ell 1}(a, n h)+(-1)^{\ell} 2-\mathcal{K}_{\ell}(n h),
$$

when the solutions $\varphi_{\ell 2}(a, n h)$ and $\vartheta_{\ell 1}(a, n h)$ of the initial value problem (1.5)-(2.1) are computed numerically at the nodes $\{n h\}_{n \in \mathbb{Z}_{N}(\lambda)}$. The reminder samples $\mathcal{U}_{\ell}^{(i)}(n h), i=$ $1, \ldots, r$, will be approximated using the values $\tilde{\mathcal{U}}_{\ell}(n h)$ through the sinc-Gaussian operator, i.e., $\mathcal{U}_{\ell}^{(i)}(n h) \approx \tilde{\mathcal{U}}_{\ell}^{(i)}(n h)$, with

$$
\tilde{\mathcal{U}}_{\ell}^{(i)}(n h)=\sum_{k \in \mathbb{Z}_{N}(\lambda)} \tilde{\mathcal{U}}_{\ell}(k h)\left\{\operatorname{sinc}\left(\pi h^{-1} \lambda-k \pi\right) \exp \left(-\frac{\alpha_{0}(\lambda-k h)^{2}}{N h^{2}}\right)\right\}_{\lambda=n h}^{(i)}
$$

where $i=1, \ldots, r$ and $h \in(0, \pi / a]$. We have restricted the parameter $h$ to be in the interval $(0, \pi / a]$ because the sinc-Gaussian operator $\left(\mathcal{G}_{0, h, N}\right)$ is defined only in this interval. The following interesting function, which is defined using the generalized sinc-Gauss operator,

$$
\widetilde{\Delta}_{\ell, N}(\lambda)(\lambda):=\mathcal{K}_{\ell}(\lambda)+\mathcal{G}_{r, h, N}\left[\tilde{\mathcal{U}}_{\ell}\right](\lambda), \quad h \in(0, \pi / a],
$$

is determined explicitly and is very close to the characteristic function $\Delta_{\ell}(\lambda)$, as we will see in Theorem 3.2 below. Therefore, the zeros of $\widetilde{\Delta}_{\ell, N}(\lambda)$ are very close to the desired zeros of $\Delta_{\ell}(\lambda)$. 
3. The error analysis. This section is devoted to investigate the error analysis associated with our method. First, we need to estimate a bound for the amplitude error associated with the generalized sinc-Gaussian operator $\mathcal{G}_{r, h, N}\left[\mathcal{U}_{\ell}\right]$. We assume that the $\widetilde{\mathcal{U}}_{\ell}^{(i)}(n h)$ are close to $\mathcal{U}_{\ell}^{(i)}(n h)$, i.e., there is $\varepsilon>0$ sufficiently small such that

$$
\sup _{n \in \mathbb{Z}_{N}(z)}\left|\widetilde{\mathcal{U}}_{\ell}^{(i)}(n h)-\mathcal{U}_{\ell}^{(i)}(n h)\right|<\varepsilon, \quad i=0, \ldots, r .
$$

We define the amplitude error associated with the operator (1.1) as follows

$$
\mathcal{A}_{r, h, N}\left[\mathcal{U}_{\ell}\right](\lambda):=\mathcal{G}_{r, h, N}\left[\mathcal{U}_{\ell}\right](\lambda)-\mathcal{G}_{r, h, N}\left[\tilde{\mathcal{U}}_{\ell}\right](\lambda) .
$$

In the following theorem, we will estimate a bound for the amplitude error $\mathcal{A}_{r, h, N}$ in the complex domain. Undoubtedly, in this paper we need the bound of the amplitude error on a real domain only because the eigenvalues of problem (1.5)-(1.6) are real, but we expect that this method will be used for boundary value problems that have complex eigenvalues.

THEOREM 3.1. Let $\sigma>0, h \in(0,(r+1) \pi / \sigma]$ and $\alpha_{r}=((r+1) \pi-h \sigma) / 2$. Assume that (3.1) holds. Then we have for $\lambda \in \mathbb{C},|\Im \lambda|<N$,

$$
\left|\mathcal{A}_{r, h, N}\left[\mathcal{U}_{\ell}\right](\lambda)\right| \leq \varepsilon C_{r, h, N}\left(1+\sqrt{N / \alpha_{r}}\right) e^{-\alpha_{r} / 4 N} e^{\left((r+1) h+\alpha_{r}\right) h^{-1}|\Im \lambda|},
$$

where $C_{r, h, N}$ is defined by

$$
C_{r, h, N}:=\frac{2}{\pi^{r+1}} \sum_{i+j+k+l=r} h^{j+1}\left|\beta_{i, j, k, l}\right| \sum_{m=0}^{\lfloor k / 2\rfloor} \frac{k !\left(2 \sqrt{\alpha_{r}}\left(h^{-1}+1\right)\right)^{k-2 m}}{m !(k-2 m) !} N^{1 / 2(k-2 m)-j-1},
$$

and the coefficients $\beta_{i, j, k, l}$ are defined in (1.3).

Proof. From the definition of the amplitude error (3.2) and using the inequality $|\sin (\lambda)| \leq$ $e^{|\Im \lambda|}$, we obtain

$$
\left|\mathcal{A}_{r, h, N}\left[\mathcal{U}_{\ell}\right](\lambda)\right| \leq \varepsilon \mathcal{L}_{r, h}(\lambda) \sum_{i+j+k+l=r}\left|\beta_{i, j, k, l}\right| \sum_{n \in \mathbb{Z}_{N}(\lambda)}\left|\frac{H_{k}\left(\frac{\sqrt{\alpha_{r}}(\lambda-n h)}{\sqrt{N} h}\right) e^{-\frac{\alpha_{r}}{N}\left(h^{-1} \lambda-n\right)^{2}}}{\left(h^{-1} \lambda-n\right)^{j+1}}\right|,
$$

where we have used estimate (3.1) and $\mathcal{L}_{r, h}(\lambda):=e^{(r+1) \pi h^{-1}|\Im \lambda|} / \pi^{r+1}$. Furthermore, we have used the fact that the coefficient $\left|\beta_{i, j, k, l}\right|$ is independent of the value $n$ and the finite sums are interchanged. The last summation of (3.4) can be bounded as

$$
\sum_{n \in \mathbb{Z}_{N}(\lambda)}\left|\frac{H_{k}\left(\frac{\sqrt{\alpha_{r}}(\lambda-n h)}{\sqrt{N} h}\right) e^{-\frac{\alpha_{r}}{N}\left(h^{-1} \lambda-n\right)^{2}}}{\left(h^{-1} \lambda-n\right)^{j+1}}\right| \leq \mathcal{S}_{k, r, h} \sum_{n \in \mathbb{Z}_{N}(\lambda)}\left|e^{-\frac{\alpha_{r}}{N}\left(h^{-1} \lambda-n\right)^{2}}\right|,
$$

where $\mathcal{R}$ is the following rectangle

$$
\mathcal{R}:=\left\{\lambda \in \mathbb{C}:\left|\left\lfloor h^{-1} \Re \lambda+1 / 2\right\rfloor-n\right| \leq N,|\Im \lambda|<N\right\}
$$

and

$$
\mathcal{S}_{k, r, h}:=\sup _{\lambda \in \mathcal{R}}\left|\frac{H_{k}\left(\frac{\sqrt{\alpha_{r}}(\lambda-n h)}{\sqrt{N} h}\right)}{\left(h^{-1} \lambda-n\right)^{j+1}}\right|
$$


It is easy to see that

$$
\sup _{\lambda \in \mathcal{R}}|\lambda-n h| \leq(1+h) N
$$

and

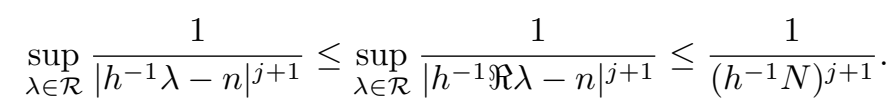

In view of (3.6), (3.7), and (1.2), we obtain

$$
\mathcal{S}_{k, r, h} \leq h^{j+1} \sum_{m=0}^{\lfloor k / 2\rfloor} \frac{k !\left(2 \sqrt{\alpha_{r}}\left(h^{-1}+1\right)\right)^{k-2 m}}{m !(k-2 m) !} N^{1 / 2(k-2 m)-j-1} .
$$

The summation in the right-hand side of (3.5) is bounded in [1, pp. 297-298] as follows

$$
\left(\sum_{n \in \mathbb{Z}_{N}(\lambda)}\left|e^{-\frac{\alpha_{r}}{N}\left(h^{-1} \lambda-n\right)^{2}}\right|^{2}\right)^{1 / 2} \leq 2\left(1+\sqrt{N / \alpha_{r}}\right) e^{-\alpha_{r} / 4 N} e^{\alpha_{r} h^{-1}|\Im \lambda|} .
$$

Combining (3.9), (3.8), (3.5), and (3.4), implies (3.3).

In the following theorem, we will prove that the function $\widetilde{\Delta}_{\ell, N}(\lambda)$, which is defined in (2.16), is an accurate approximation to the characteristic function $\Delta_{\ell}(\lambda)$.

THEOREM 3.2. Let $N \in \mathbb{N}, \lambda \in \mathbb{R}$ and assume that condition (3.1) holds. Then we have the following estimate

$$
\left|\Delta_{\ell}(\lambda)-\widetilde{\Delta}_{\ell, N}(\lambda)\right| \leq T_{N, k, h}(\lambda)+A_{\varepsilon, h, N}
$$

where $\Delta_{\ell}$ is the characteristic function of the problem (1.5)-(1.6) and $\widetilde{\Delta}_{\ell, N}$ is defined in (2.16). The functions $T_{N, k, h}$ and $A_{\varepsilon, h, N}$ are defined by

$$
\begin{aligned}
T_{N, k, h}(\lambda) & :=2^{r}\left|\sin ^{r+1}\left(h^{-1} \pi \lambda\right)\right|\|f\|_{\infty} \frac{e^{-\alpha_{r} N}}{\pi \sqrt{\alpha_{r} N}}, \\
A_{\varepsilon, h, N} & :=\varepsilon C_{r, h, N}\left(1+\sqrt{N / \alpha_{r}}\right) e^{-\alpha_{r} / 4 N}
\end{aligned}
$$

where $h \in(0, \pi / a], \alpha_{r}=((r+1) \pi-h a) / 2$. Moreover $\widetilde{\Delta}_{\ell, N} \rightarrow \Delta_{\ell}$ uniformly on $\mathbb{R}$ when $\varepsilon \rightarrow 0$ and $N \rightarrow \infty$.

Proof. According to (2.16) and (2.12), we have

$$
\left|\Delta_{\ell}(\lambda)-\widetilde{\Delta}_{\ell, N}(\lambda)\right| \leq\left|\mathcal{U}_{\ell}(\lambda)-\mathcal{G}_{r, h, N}\left[\mathcal{U}_{\ell}\right](\lambda)\right|+\left|\mathcal{G}_{r, h, N}\left[\mathcal{U}_{\ell}\right](\lambda)-\mathcal{G}_{r, h, N}\left[\tilde{\mathcal{U}}_{\ell}\right](\lambda)\right|
$$

Since $\mathcal{U}_{\ell} \in B_{a}^{\infty}$, we can approximate $\mathcal{U}_{\ell}$ by the generalized sampling operator (1.1) and we have (cf. (1.4))

$$
\left|\mathcal{U}_{\ell}(\lambda)-\mathcal{G}_{r, h, N}\left[\mathcal{U}_{\ell}\right](\lambda)\right| \leq T_{N, k, h}(\lambda), \quad \lambda \in \mathbb{R}
$$

where $T_{N, k, h}$ is defined by (3.11). Since condition (3.1) holds, we have (cf. (3.3))

$$
\left|\mathcal{G}_{r, h, N}\left[\mathcal{U}_{\ell}\right](\lambda)-\mathcal{G}_{r, h, N}\left[\tilde{\mathcal{U}}_{\ell}\right](\lambda)\right| \leq A_{\varepsilon, h, N},
$$


where $A_{\varepsilon, h, N}$ is defined by (3.12). Combining (3.15), (3.14), and (3.13), implies (3.10). In view of (3.11) and (3.12), the right-hand side of (3.10) goes to zero uniformly on $\mathbb{R}$ when $N \rightarrow \infty$ and $\varepsilon \rightarrow 0$. Therefore, $\widetilde{\Delta}_{\ell, N} \rightarrow \Delta_{\ell}$ uniformly on $\mathbb{R}$ when $\varepsilon \rightarrow 0$ and $N \rightarrow \infty$.

Now, we will use the bound in (3.10) to estimate the absolute error $\left|\lambda_{\ell}^{*}-\lambda_{\ell, N}\right|$ when $\lambda_{\ell}^{*}$ is the exact eigenvalue of the problem (1.5)-(1.6) and $\lambda_{\ell, N}$ is the zero of the function $\tilde{\Delta}_{\ell, N}(\lambda)$.

THEOREM 3.3. Let $\lambda_{\ell}^{*}$ be an eigenvalue of (1.5)-(1.6) of multiplicity $\nu(\nu=1$ or 2$)$ and denote by $\lambda_{\ell, N}$ the corresponding approximation. Then, for $\lambda_{\ell, N} \in \mathbb{R}$, we have the following estimate

$$
\left|\lambda_{\ell}^{*}-\lambda_{\ell, N}\right|<\left(\frac{\nu !\left(T_{N, k, h}\left(\lambda_{\ell, N}\right)+A_{\varepsilon, h, N}\right)}{\inf _{\zeta \in \mathcal{I}_{\ell, N}}\left|\Delta_{\ell}^{(\nu)}(\zeta)\right|}\right)^{1 / \nu}
$$

where $\mathcal{I}_{\ell, N}:=\left[\min \left\{\lambda_{\ell}^{*}, \lambda_{\ell, N}\right\}, \max \left\{\lambda_{\ell}^{*}, \lambda_{\ell, N}\right\}\right]$. Moreover, $\left|\lambda^{*}-\lambda_{N, k}\right| \longrightarrow 0$ when $N \rightarrow \infty$ and $\varepsilon \rightarrow 0$.

Proof. Replacing $\lambda$ by $\lambda_{\ell, N}$ in (3.10), we obtain

$$
\left|\Delta_{\ell}\left(\lambda_{\ell, N}\right)-\Delta_{\ell}\left(\lambda_{\ell}^{*}\right)\right|<T_{N, k, h}\left(\lambda_{\ell, N}\right)+A_{\varepsilon, h, N},
$$

where we have used $\widetilde{\Delta}_{\ell, N}\left(\lambda_{\ell, N}\right)=\Delta_{\ell}\left(\lambda_{\ell}^{*}\right)=0$. Since $\lambda_{\ell}^{*}$ is a zero of $\Delta_{\ell}(\lambda)$ with multiplicity of $\nu$, we have from the reminder term of the Taylor expansion

$$
\Delta_{\ell}\left(\lambda_{\ell}^{*}\right)-\Delta_{\ell}\left(\lambda_{\ell, N}\right)=\frac{\left(\lambda_{\ell}^{*}-\lambda_{\ell, N}\right)^{\nu}}{\nu !} \Delta_{\ell}^{(\nu)}(\zeta)
$$

for some $\zeta \in \mathcal{I}_{\ell, N}$. From (3.18) and (3.17), we get

$$
\left|\lambda_{\ell}^{*}-\lambda_{\ell, N}\right|^{\nu}\left|\Delta_{\ell}^{(\nu)}(\zeta)\right|<\nu !\left(T_{N, k, h}\left(\lambda_{\ell, N}\right)+A_{\varepsilon, h, N}\right), \quad \zeta \in \mathcal{I}_{\ell, N}
$$

Since the eigenvalue $\lambda_{\ell}^{*}$ is of multiplicity $\nu$, we have $\Delta_{\ell}^{(\nu)}(\zeta)\left(\lambda_{\ell}^{*}\right) \neq 0$. Therefore, for sufficiently large $N$, we have

$$
\inf _{\zeta \in \mathcal{I}_{\ell, N}}\left|\Delta_{\ell}^{(\nu)}(\zeta)\right|>0
$$

and hence dividing by the left-hand side of (3.19), we obtain (3.16). The remaining part of the proof follows from the fact that $T_{N, k, h} \rightarrow 0$ as $N \rightarrow \infty$, and $A_{\varepsilon, h, N} \rightarrow 0$ as $\varepsilon \rightarrow 0$.

4. Illustrative examples. This section includes four examples to illustrate the advantages of the proposed method. The Dirac systems in Examples 4.1, 4.4 are semi-periodic, while those in Examples 4.2, 4.3 are periodic. In Example 4.3, the eigenvalues in the interval $[-1,4]$ are simple. The eigenvalues in the other examples are double except for Example 4.4, where one eigenvalue of is simple. We will compute the eigenvalues using the operator $\mathcal{G}_{r, h, N}$ with $r=0,1,2,3$. As predicted by the error estimates, the precision increases when $N$ and $h$ are fixed and $r$ increases, with the additional cost that the samples of the derivatives of functions need to be calculated using the samples of the function itself; cf. (2.15). In all examples, it is a simple task to compute the characteristic function, $\Delta_{\ell}(\lambda)$ explicitly, except in Example 4.3 where it cannot be computed in closed form. Therefore, the amplitude error only appears in 
Example 4.3. In all examples, we denote the eigenvalues and their approximations by $\lambda_{k}$ and $\lambda_{k, N}$ respectively.

EXAMPLE 4.1. Consider the semi-periodic Dirac system

$$
\begin{aligned}
u_{2}^{\prime}(x) & +x u_{1}(x)=\lambda u_{1}(x), \\
-u_{1}^{\prime}(x) & +x u_{2}(x)=\lambda u_{2}(x), \quad x \in[0,4], \\
u_{1}(0) & =-u_{1}(4), \quad u_{2}(0)=-u_{2}(4) .
\end{aligned}
$$

Here $a=4, \ell=2$, and $p_{1}(x)=p_{2}(x)=x$. The characteristic function is

$$
\Delta_{2}(\lambda)=2-2 \cos (2(4-2 \lambda)),
$$

and the exact eigenvalues are $\lambda_{k}=\frac{4-\pi k}{2}, k \in \mathbb{Z}$. Since all the eigenvalues of this problem satisfy condition (2.3), they are all double eigenvalues. According to (2.14), after calculating the function $\mathcal{K}_{2}$, we get

$$
\Delta_{2, N}(\lambda)=2-2 \cos (4 \lambda)+\mathcal{G}_{r, h, N}\left[\mathcal{U}_{2}\right](\lambda) .
$$

In Table 4.1, we give the approximate eigenvalues obtained by the generalized sinc-Gaussian sampling technique, with $N=7, h=0.7$, and different values of $r$. Table 4.2 reports the absolute error $\left|\lambda_{k}-\lambda_{k, N}\right|$ for $r=0,1,2,3$. Figures 4.1 and 4.2 illustrate the difference between the function $\Delta_{2}(\lambda)$ and its approximation $\Delta_{2, N}(\lambda)$, with $r=0,2$, in the interval $[-2,6]$.

TABLE 4.1

The eigenvalues with $N=7$ and $h=0.7$.

\begin{tabular}{c|c|c|c|c}
$k$ & $\mathcal{G}_{0, h, N}$ & $\mathcal{G}_{1, h, N}$ & $\mathcal{G}_{2, h, N}$ & $\mathcal{G}_{3, h, N}$ \\
\hline-2 & 5.11343254829677 & 5.14159196109544 & 5.141592653560071 & 5.141592653589792 \\
-1 & 3.52587616111651 & 3.57079634599230 & 3.570796326799396 & 3.570796326794903 \\
0 & 1.99268698750402 & 1.99999950376364 & 1.999999999992704 & 2.000000000000004 \\
1 & 0.44747045787135 & 0.42920436334952 & 0.429203673230348 & 0.429203673205104 \\
2 & -1.16420081558931 & -1.14159321960092 & -1.141592653617637 & -1.141592653589794
\end{tabular}

TABLE 4.2

The absolute error $\left|\lambda_{k}-\lambda_{k, N}\right|$ with $N=7$ and $h=0.7$.

\begin{tabular}{c|c|c|c|c}
$\lambda_{k}$ & $\mathcal{G}_{0, h, N}$ & $\mathcal{G}_{1, h, N}$ & $\mathcal{G}_{2, h, N}$ & $\mathcal{G}_{3, h, N}$ \\
\hline$\lambda_{-2}$ & $2.81601 \times 10^{-2}$ & $6.92494 \times 10^{-7}$ & $2.9722 \times 10^{-11}$ & $8.88178 \times 10^{-16}$ \\
$\lambda_{-1}$ & $4.49202 \times 10^{-2}$ & $1.91974 \times 10^{-8}$ & $4.49907 \times 10^{-12}$ & $6.66134 \times 10^{-15}$ \\
$\lambda_{0}$ & $7.31301 \times 10^{-3}$ & $4.96236 \times 10^{-7}$ & $7.29594 \times 10^{-12}$ & $4.44089 \times 10^{-15}$ \\
$\lambda_{1}$ & $1.82668 \times 10^{-2}$ & $6.90144 \times 10^{-7}$ & $2.52441 \times 10^{-11}$ & $6.10623 \times 10^{-16}$ \\
$\lambda_{2}$ & $2.26082 \times 10^{-2}$ & $5.66011 \times 10^{-7}$ & $2.78437 \times 10^{-11}$ & $8.88178 \times 10^{-16}$
\end{tabular}

EXAMPLE 4.2. The boundary value problem

$$
\begin{aligned}
u_{2}^{\prime}(x) & +x^{2} u_{1}(x)=\lambda u_{1}(x), \\
-u_{1}^{\prime}(x) & +x^{2} u_{2}(x)=\lambda u_{2}(x), \quad x \in[0, \pi], \\
u_{1}(0) & =u_{1}(\pi), \quad u_{2}(0)=u_{2}(\pi),
\end{aligned}
$$




\section{ETNA}

Kent State University and

Johann Radon Institute (RICAM)

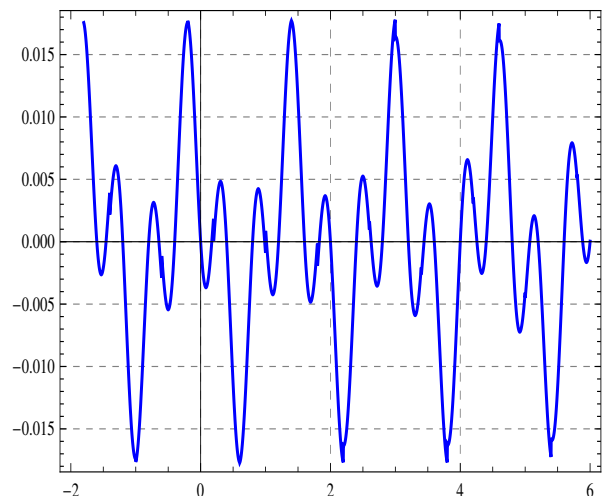

FIG. 4.1. $\Delta_{2}(\lambda)-\Delta_{2, N}(\lambda)$ for $r=0, N=$ 5 , and $h=0.4$

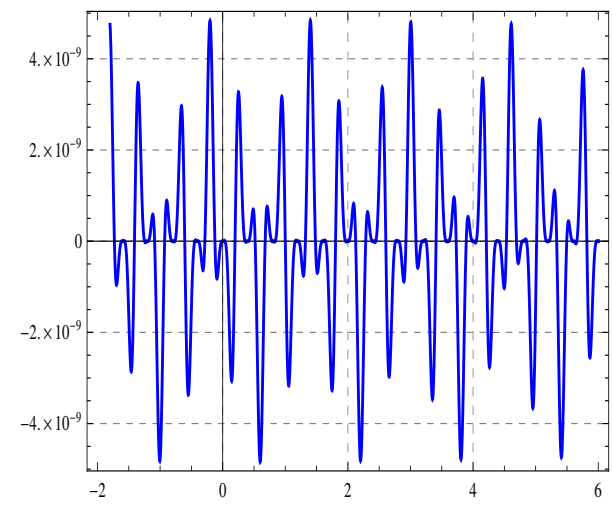

FIG. 4.2. $\Delta_{2}(\lambda)-\Delta_{2, N}(\lambda)$ for $r=2, N=$ 5 , and $h=0.4$.

is a periodic Dirac system with $a=\pi, \ell=1$, and $p_{1}(x)=p_{2}(x)=x^{2}$. Here the characteristic function is

$$
\Delta_{1}(\lambda)=2 \cos \left(\frac{\pi}{3}\left(\pi^{2}-3 \lambda\right)\right)-2
$$

Hence the exact eigenvalues are $\lambda_{k}=\frac{\pi^{2}-6 k}{3}, k \in \mathbb{Z}$. Since $\lambda_{k}, k \in \mathbb{Z}$, satisfy the condition (2.3), all eigenvalues of this problem are double. The function $\Delta_{1, N}(\lambda)$ is

$$
\Delta_{1, N}(\lambda)=2 \cos (\pi \lambda)-2+\mathcal{G}_{r, h, N}\left[\mathcal{U}_{1}\right](\lambda) .
$$

Table 4.3 list the approximate eigenvalues of this problem with $N=7, h=0.7$, and $r=0,1,2,3$. In Table 4.4, we display the absolute error for various choices of $r$. The plots of $\Delta_{1}(\lambda)-\Delta_{1, N}(\lambda)$ with $N=5, h=0.5, r=1$, and $N=5, h=0.5, r=3$, are shown in Figures 4.3 and 4.4 , respectively, for the interval $[-2,8]$.

TABLE 4.3

The eigenvalues with $N=7$ and $h=0.8$.

\begin{tabular}{c|c|c|c|c}
$k$ & $\mathcal{G}_{0, h, N}$ & $\mathcal{G}_{1, h, N}$ & $\mathcal{G}_{2, h, N}$ & $\mathcal{G}_{3, h, N}$ \\
\hline-2 & 7.29146784300787 & 7.28986836898996 & 7.28986813369914 & 7.2898681336964835 \\
-1 & 5.29710113074795 & 5.28986849957303 & 5.28986813371427 & 5.2898681336964590 \\
0 & 3.29047941860795 & 3.28986836862110 & 3.28986813369913 & 3.2898681336964680 \\
1 & 1.29710113074795 & 1.28986849957303 & 1.28986813371426 & 1.2898681336964537 \\
2 & -0.70952058139205 & -0.71013163137890 & -0.71013186630088 & -0.7101318663035477
\end{tabular}

TABLE 4.4

The absolute error $\left|\lambda_{k}-\lambda_{k, N}\right|$ with $N=7$ and $h=0.8$.

\begin{tabular}{c|c|c|c|c}
$\lambda_{k}$ & $\mathcal{G}_{0, h, N}$ & $\mathcal{G}_{1, h, N}$ & $\mathcal{G}_{2, h, N}$ & $\mathcal{G}_{3, h, N}$ \\
\hline$\lambda_{-2}$ & $1.59971 \times 10^{-3}$ & $2.35294 \times 10^{-7}$ & $2.68408 \times 10^{-12}$ & $3.01981 \times 10^{-14}$ \\
$\lambda_{-1}$ & $7.23300 \times 10^{-3}$ & $3.65877 \times 10^{-7}$ & $1.78124 \times 10^{-11}$ & $6.21725 \times 10^{-15}$ \\
$\lambda_{0}$ & $6.11285 \times 10^{-4}$ & $2.34925 \times 10^{-7}$ & $2.67564 \times 10^{-12}$ & $1.55431 \times 10^{-14}$ \\
$\lambda_{1}$ & $7.23300 \times 10^{-3}$ & $3.65877 \times 10^{-7}$ & $1.78098 \times 10^{-11}$ & $1.33227 \times 10^{-15}$ \\
$\lambda_{2}$ & $6.11285 \times 10^{-4}$ & $2.34925 \times 10^{-7}$ & $2.66343 \times 10^{-12}$ & $2.22045 \times 10^{-16}$
\end{tabular}




\section{ETNA}

Kent State University and

Johann Radon Institute (RICAM)

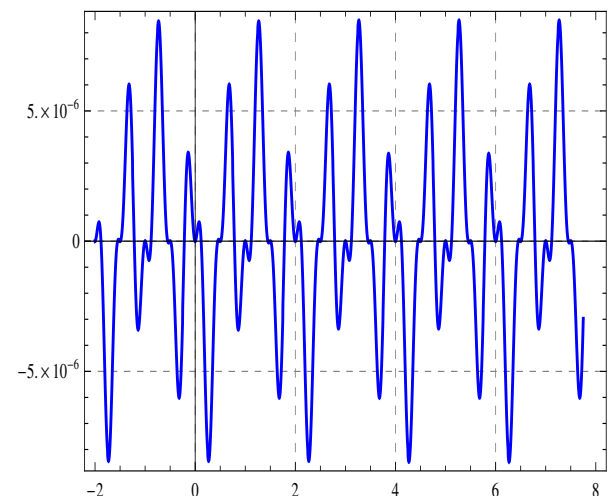

FIG. 4.3. $\Delta_{1}(\lambda)-\Delta_{1, N}(\lambda)$ for $r=1, N=$ 5 , and $h=0.5$.

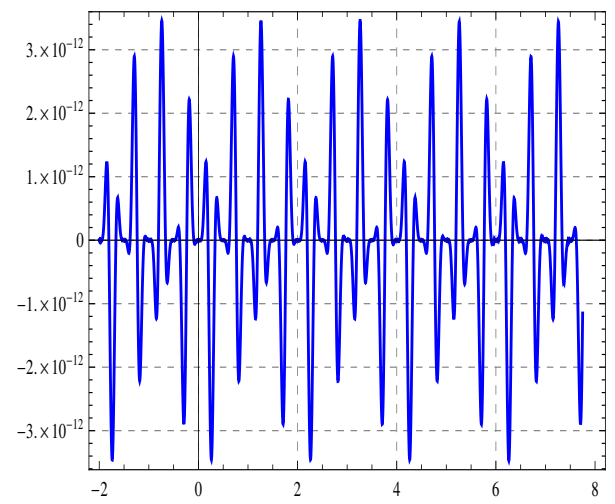

FIG. 4.4. $\Delta_{1}(\lambda)-\Delta_{1, N}(\lambda)$ for $r=3, N=$ 5 , and $h=0.5$.

EXAMPLE 4.3. The boundary value problem

$$
\begin{aligned}
u_{2}^{\prime}(x) & +u_{1}(x)=\lambda u_{1}(x), \\
-u_{1}^{\prime}(x) & +x u_{2}(x)=\lambda u_{2}(x), \quad x \in[0,3], \\
u_{1}(0) & =u_{1}(3), \quad u_{2}(0)=u_{2}(3),
\end{aligned}
$$

is a special case of the periodic problem when $a=3, \ell=1, p_{1}(x)=1$, and $p_{2}(x)=x$. The characteristic function of this problem cannot be computed in a closed form and, thus, the amplitude error appears. It is given as a combination of four varieties of Airy functions. The eigenvalues of this problem are only simple. Here

$$
\widetilde{\Delta}_{1, N}(\lambda)=2 \cos (3 \lambda)-2+\mathcal{G}_{r, h, N}\left[\widetilde{\mathcal{U}}_{1}\right](\lambda) .
$$

As in the previous examples, Tables 4.5, 4.6, and Figures 4.5, 4.6, illustrate the application of our technique to this problem and the effect of the parameter $r$.

TABLE 4.5

The eigenvalues with $N=6$ and $h=0.6$.

\begin{tabular}{c|c|c|c|c}
$k$ & $\mathcal{G}_{0, h, N}$ & $\mathcal{G}_{1, h, N}$ & $\mathcal{G}_{2, h, N}$ & $\mathcal{G}_{3, h, N}$ \\
\hline-2 & -0.989307528897929 & -0.996849937695628 & -0.996850335848082 & -0.996850335861101 \\
-1 & -0.781316586278270 & -0.774237301037988 & -0.774236951398153 & -0.774236951392367 \\
0 & 1.002087607417726 & 1.000000023208921 & 0.999999999986544 & 0.999999999999996 \\
1 & 1.447845400808394 & 1.449598042862364 & 1.449598100700345 & 1.449598100695762 \\
2 & 3.260293987328006 & 3.254026906173752 & 3.254026412457459 & 3.254026412407036 \\
3 & 3.538940501410063 & 3.540787891656990 & 3.540787887151272 & 3.540787887149195
\end{tabular}

EXAMPLE 4.4. The semi-periodic Dirac system

$$
\begin{gathered}
u_{2}^{\prime}(x)-u_{1}(x)=\lambda u_{1}(x), \\
-u_{1}^{\prime}(x)+u_{2}(x)=\lambda u_{2}(x), \quad x \in[0,4], \\
u_{1}(0)=-u_{1}(4), \quad u_{2}(0)=-u_{2}(4),
\end{gathered}
$$

has $a=4, \ell=2, p_{1}(x)=-1$, and $p_{2}(x)=1$. The characteristic function is

$$
\Delta_{2}(\lambda)=2-2 \cos \left(4 \sqrt{\lambda^{2}-1}\right) .
$$




\section{ETNA}

Kent State University and

Johann Radon Institute (RICAM)

TABLE 4.6

The absolute error $\left|\lambda_{k}-\lambda_{k, N}\right|$ with $N=6$ and $h=0.6$.

\begin{tabular}{c|c|c|c|c|c}
$\lambda_{k}$ & Exact $\lambda_{k}$ & $\mathcal{G}_{0, h, N}$ & $\mathcal{G}_{1, h, N}$ & $\mathcal{G}_{2, h, N}$ & $\mathcal{G}_{3, h, N}$ \\
\hline$\lambda_{-2}$ & -0.996850335861097 & $7.5428 \times 10^{-3}$ & $3.9817 \times 10^{-7}$ & $1.3015 \times 10^{-11}$ & $4.3299 \times 10^{-15}$ \\
$\lambda_{-1}$ & -0.774236951392368 & $7.0796 \times 10^{-3}$ & $3.4965 \times 10^{-7}$ & $5.7858 \times 10^{-12}$ & $2.2205 \times 10^{-16}$ \\
$\lambda_{0}$ & 1.000000000000000 & $2.0876 \times 10^{-3}$ & $2.3209 \times 10^{-8}$ & $1.3456 \times 10^{-11}$ & $4.1078 \times 10^{-15}$ \\
$\lambda_{1}$ & 1.449598100695761 & $1.7527 \times 10^{-3}$ & $5.7833 \times 10^{-8}$ & $4.5841 \times 10^{-12}$ & $8.8818 \times 10^{-16}$ \\
$\lambda_{2}$ & 3.254026412407030 & $6.2676 \times 10^{-3}$ & $4.9377 \times 10^{-7}$ & $5.0429 \times 10^{-11}$ & $6.6613 \times 10^{-15}$ \\
$\lambda_{3}$ & 3.540787887149186 & $1.8474 \times 10^{-3}$ & $4.5078 \times 10^{-9}$ & $2.0854 \times 10^{-12}$ & $9.3259 \times 10^{-15}$
\end{tabular}

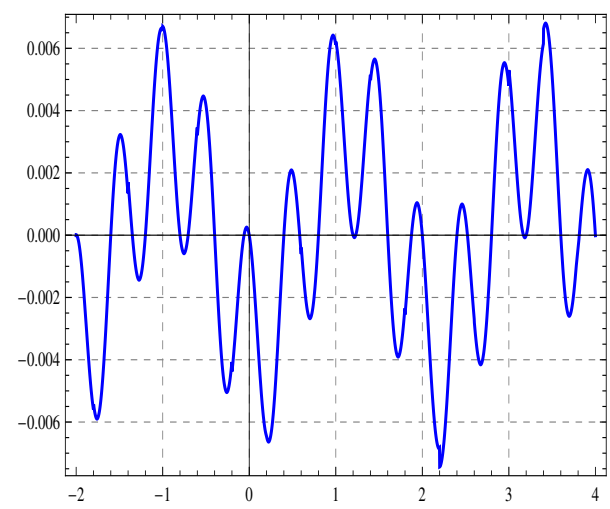

FIG. 4.5. $\Delta_{1}(\lambda)-\widetilde{\Delta}_{1, N}(\lambda)$ for $r=0, N=$ 5 , and $h=0.4$.

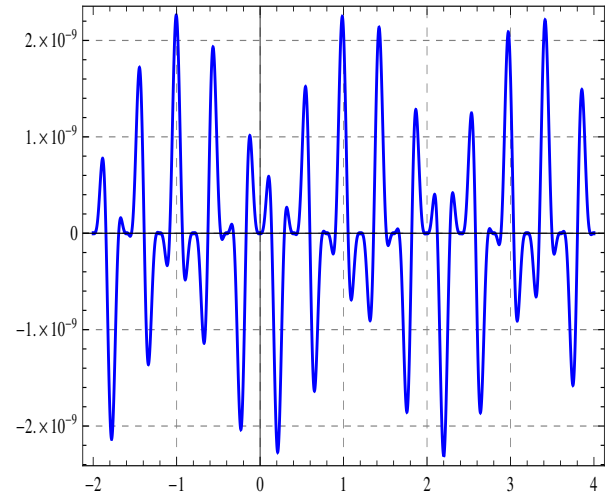

FIG. 4.6. $\Delta_{1}(\lambda)-\widetilde{\Delta}_{1, N}(\lambda)$ for $r=2, N=$ 5 , and $h=0.4$.

The eigenvalues are exactly $\lambda_{k}^{2}=\frac{(\pi k)^{2}+4}{4}, \quad k \in \mathbb{Z}$. The only simple eigenvalue of this problem is at $k=0$. In this example,

$$
\Delta_{2, N}(\lambda)=2-2 \cos (4 \lambda)++\mathcal{G}_{r, h, N}\left[\mathcal{U}_{2}\right](\lambda) .
$$

We report the exact eigenvalues and their approximations in Tables 4.7 and 4.8. Here Figures 4.7 and 4.8 illustrate the behaviour of $\Delta_{2}(\lambda)-\Delta_{2, N}(\lambda)$ when $N=5, h=0.4$, and $r=1,3$, in the interval $[0,7]$.

TABLE 4.7

The eigenvalues with $N=6$ and $h=0.7$.

\begin{tabular}{c|c|c|c|c}
$k$ & $\mathcal{G}_{0, h, N}$ & $\mathcal{G}_{1, h, N}$ & $\mathcal{G}_{2, h, N}$ & $\mathcal{G}_{3, h, N}$ \\
\hline 0 & 1.001632496378207 & 1.000000145431083 & 1.000000000010789 & 1.000000000000000 \\
1 & 1.864138728327801 & 1.862096874886582 & 1.862095889331847 & 1.862095889118621 \\
2 & 3.297725071148114 & 3.296909099124057 & 3.296908309514440 & 3.296908309475604 \\
3 & 4.798608651402407 & 4.817323383723065 & 4.817323935771017 & 4.817323935802022 \\
4 & 6.349446510761929 & 6.362265174718638 & 6.362265131586696 & 6.362265131567328
\end{tabular}

5. Conclusions. This work is devoted to constructing a new sampling method to approximate the eigenvalues of the periodic (semi-periodic) Dirac system of differential equations problem. This method is built by using the generalized sinc-Gaussian sampling operator $\mathcal{G}_{r, h, N}$, which was established by Asharabi in 2016. This operator has a convergence rate of exponential type, i.e., $\mathrm{e}^{-\alpha_{r} N} / \sqrt{N}, \alpha_{r}=(r+1) \pi-\sigma h$. The method is based on constructing 


\section{ETNA}

Kent State University and

Johann Radon Institute (RICAM)

GENERALIZED SINC-GAUSSIAN FOR EIGENVALUES OF THE DIRAC SYSTEM

TABLE 4.8

The absolute error $\left|\lambda_{k}-\lambda_{k, N}\right|$ with $N=6$ and $h=0.7$.

\begin{tabular}{c|c|c|c|c}
$\lambda_{k}$ & $\mathcal{G}_{0, h, N}$ & $\mathcal{G}_{1, h, N}$ & $\mathcal{G}_{2, h, N}$ & $\mathcal{G}_{3, h, N}$ \\
\hline$\lambda_{0}$ & $1.63250 \times 10^{-3}$ & $1.45431 \times 10^{-7}$ & $1.07894 \times 10^{-11}$ & $2.22045 \times 10^{-16}$ \\
$\lambda_{1}$ & $2.04284 \times 10^{-3}$ & $9.85768 \times 10^{-7}$ & $2.13261 \times 10^{-10}$ & $3.44169 \times 10^{-14}$ \\
$\lambda_{2}$ & $8.16762 \times 10^{-4}$ & $7.89648 \times 10^{-7}$ & $3.88245 \times 10^{-11}$ & $1.15463 \times 10^{-14}$ \\
$\lambda_{3}$ & $1.87153 \times 10^{-2}$ & $5.52079 \times 10^{-7}$ & $3.10019 \times 10^{-11}$ & $2.66454 \times 10^{-15}$ \\
$\lambda_{4}$ & $1.28186 \times 10^{-2}$ & $4.31513 \times 10^{-8}$ & $1.93685 \times 10^{-11}$ & $8.88178 \times 10^{-16}$
\end{tabular}

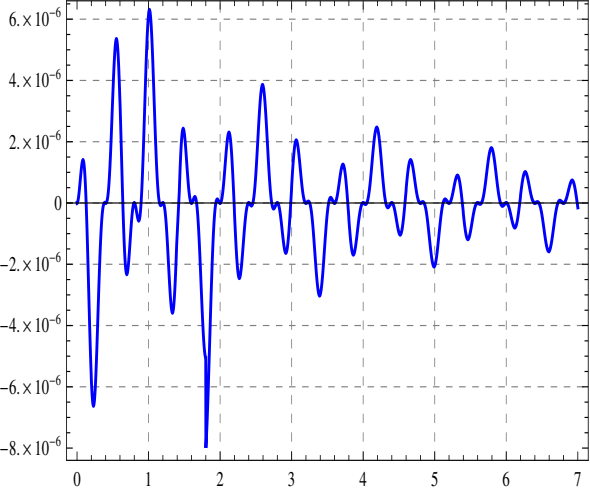

FIG. 4.7. $\Delta_{2}(\lambda)-\Delta_{2, N}(\lambda)$ for $r=1, N=$ 5 , and $h=0.4$.

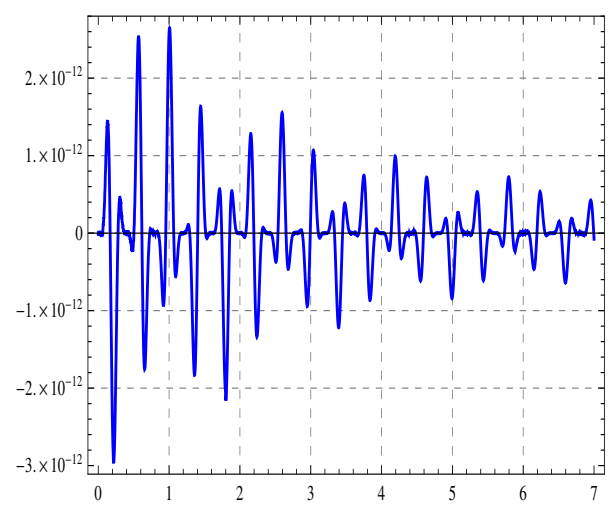

FIG. 4.8. $\Delta_{2}(\lambda)-\Delta_{2, N}(\lambda)$ for $r=3, N=$ 5 , and $h=0.4$.

an entire function $\widetilde{\Delta}_{\ell, N}(\lambda)$, using the generalized sinc-Gaussian sampling operator, which is very close to the characteristic function $\Delta_{\ell}(\lambda)$. The zeros of $\widetilde{\Delta}_{\ell, N}(\lambda)$ closely approximate the zeros of $\Delta_{\ell}(\lambda)$. The method depends upon three parameters, $r, h, N$, that play a pivotal role for the accuracy. The accuracy of the method increases when the parameters $r, N$ are fixed and $h$ is decreasing, without additional cost except that the function is approximated on a smaller domain. Furthermore, the precision improves when $N$ and $h$ are fixed and $r$ increases, with the additional cost that the samples of the derivatives of the function need to be calculated using the samples of the function itself; cf. (2.15). This method generalizes the well-known sampling methods (sinc-Gaussian and Hermite-Gauss methods). To investigate the error analysis of this method, it is necessary to study the truncation error and the amplitude error associated to the generalized sinc-Gaussian sampling operator. The numerical examples show a strong compliance with our theoretical analysis.

\section{REFERENCES}

[1] M. H. ANNABy AND R. M. AshaRABI, Computing eigenvalues of boundary-value problems using sincGaussian method, Sampl. Theory Signal Image Process., 7 (2008), pp. 293-311.

[2] M. H. Annaby And M. M. Tharwat, On the computation of the eigenvalues of Dirac systems, Calcolo, 49 (2012), pp. 221-240.

[3] - Sinc-based computations of eigenvalues of Dirac systems, BIT, 47 (2007), pp. 699-713.

[4] R. M. AsHARABI, Generalized sinc-Gaussian sampling involving derivatives, Numer. Algorithms, 73 (2016), pp. 1055-1072.

[5] - Approximating eigenvalues of boundary value problems by using the Hermite-Gauss sampling method, Electron. Trans. Numer. Anal., 46 (2017), pp. 359-374.

http://etna.ricam.oeaw.ac.at/vol.46.2017/pp359-374.dir/pp359-374.pdf 
[6] R. M. Asharabi and J. Prestin, A modification of Hermite sampling with a Gaussian multiplier, Numer. Funct. Anal. Optim., 36 (2015), pp. 419-437.

[7] R. M. Asharabi And M. M. Tharwat, Approximating eigenvalues of Dirac system with discontinuities at several points using Hermite-Gauss method, Numer. Algorithms, 76 (2017), pp. 655-673.

[8] G. R. GRozeV AND Q. I. RAHMAn, Reconstruction of entire functions from irregularly spaced sample points, Canad. J. Math., 48 (1996), pp. 777-793.

[9] B. M. LeVitAn AND I. S. SARgSJAn, Introduction to Spectral Theory: Selfadjoint Ordinary Differential Operators, American Mathematical Society, Providence, 1975.

[10] - Sturm-Liouville and Dirac Operators, Kluwer Academic, Dordrecht, 1991.

[11] L. QIAN, On the regularized Whittaker-Kotel'nikov-Shannon sampling formula, Proc. Amer. Math. Soc., 131 (2002), pp. 1169-1176.

[12] L. QIAN AND D. B. CREAMER, A modification of the sampling series with a Gaussian multiplier, Sampl. Theory Signal Image Process., 5 (2006), pp. 1-19.

[13] G. SchmeISSER AND F. STENGER, Sinc approximation with a Gaussian multiplier, Sampl. Theory Signal Image Process., 6 (2007), pp. 199-221.

[14] C. E. SHIN, Generalized Hermite interpolation and sampling theorem involving derivatives, Commun. Korean Math. Soc., 17 (2002), pp. 731-740.

[15] J. Voss, Irregular Sampling: Error Analysis, Applications and Extensions, Mitt. Math. Sem. Giessen 238, Giessen, 1999.

[16] A. I. ZAYED, Advances in Shannon's Sampling Theory, CRC Press, Baco Raton, 1993. 